Haim Abramovich

Intelligent Materials and Structures 


\section{Also of Interest}

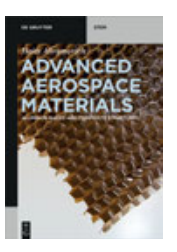

Advanced Aerospace Materials.

Aluminum-Based and Composite Structures

Abramovich, 2019

ISBN 978-3-11-053756-7, e-ISBN 978-3-11-053757-4

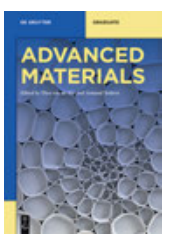

Advanced Materials

van de Ven, Soldera (Eds.), 2019

ISBN 978-3-11-053765-9, e-ISBN 978-3-11-053773-4

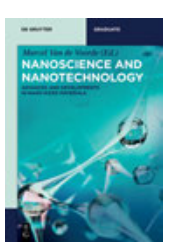

Nanoscience and Nanotechnology.

Advances and Developments in Nano-sized Materials Van de Voorde (Ed.), 2018

ISBN 978-3-11-054720-7, e-ISBN 978-3-11-054722-1

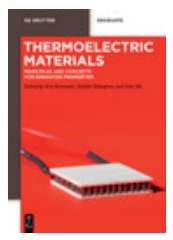

Thermoelectric Materials.

Principles and Concepts for Enhanced Properties

Kurosaki, Takagiwa, Shi 2020

ISBN 978-3-11-059648-9, e-ISBN 978-3-11-059652-6 
Haim Abramovich

Intelligent

Materials and

Structures

2nd Edition

DE GRUYTER 


\section{Authors}

Prof. Dr. Haim Abramovich

Aerospace Structural Laboratory

Technion-Israel Inst. of Technology

Technion City

32000 Haifa

Israel

ISBN 978-3-11-072669-5

e-ISBN (PDF) 978-3-11-072670-1

e-ISBN (EPUB) 978-3-11-072622-0

Library of Congress Control Number: 2021938034

Bibliographic information published by the Deutsche Nationalbibliothek

The Deutsche Nationalbibliothek lists this publication in the Deutsche Nationalbibliografie; detailed bibliographic data are available on the Internet at http://dnb.dnb.de.

(C) 2021 Walter de Gruyter GmbH, Berlin/Boston

Cover image: ThomasVogel/iStock/Getty Images Plus

Typesetting: Integra Software Services Pvt. Ltd.

Printing and binding: $\mathrm{CPI}$ books $\mathrm{GmbH}$, Leck

www.degruyter.com 